\title{
Lecturas y «deslecturas»: Mallarmé, Darío y la teoría de la misprision de Harold Bloom
}

\author{
BERNARD J. McGUIRK \\ University of Nottingham
}

\begin{abstract}
I do not believe that poetic influence is simply something that happens, that it is just the process by which ideas and images are transmitted from earlier to later poets. On that view, whether or not influence causes anxiety in the later poet is a matter of temperament and circumstance. Poetic influence thus reduces to source-study, of the kind perfomed upon Coleridge by Lowes and later scholars. Coleridge was properly scornful of such study, and I think most critics learn how barren an enterprise it turns out to be. I myself have no use for it as such, and what I mean by the study of poetic influence turns source-study inside out.
\end{abstract}

Harold Blomm ${ }^{1}$

El propósito del presente estudio no es, en primera instancia, demostrar que, en un caso dado, Mallarmé constituye para Darío una fuente literaria, a pesar de que el soneto "Yo persigo una forma» tiene como fecha el año 1900, momento en que Darío estaba en París «donde conoce el mundo literario y el de la bohemia» ${ }^{2}$. Esto sería tan sólo una invitación a emprender lo que Tzvetan Todorov describe, con cierto desprecio, como la crítica etiológica, es decir, aquella en que «el autor se

I Figures of Capable Imagination, New York, Seabury, 1976, p. 9.

2 Rubén Darío, Azul. Prosas profanas, edición, estudio y notas de Andrew P. Debicki y Michael J. Doudoroff, Madrid, Ediciones Alhambra, 1985, p. 25. 
pregunta qué puede haber conducido a Rimbaud a expresarse de tal o cual manera» o «en vez de una transparencia referencial encontramos nada más una transparencia orientada hacia el poeta, cuyo texto no es considerado como una expresión sino como una especie de síntoma» ${ }^{3}$. De manera semejante, la opinión acertada de Ignacio Zuleta de que «Yo persigo una forma», «como cierre del libro [...] alude de modo [...] directo a la vocación de decir lo indecible» ${ }^{4}$, apunta obviamente al culto paralelo mallarmeano de l'indicible, e invita al crítico vigilante a hacer una comparación de los respectivos poemas-cierre de Prosas profanas y Poesíes.

El hecho es que la investigación sobre Darío ha sido tocada en los aspectos tanto positivos como negativos del método del cual se trata aquí. Una fuente literaria, por mucho que haya sido descubierta o demostrada, queda, dentro del estudio de las influencias, a un nivel de interés limitado, es decir, constituye un mero hecho. Lo que le interesa más a Harold Bloom es la cuestión de cómo funciona, y por qué, la influencia.

Así, en dos libros relativamente recientes, The Anxiety of Influence (1973) y A Map of Misreading (1975) ${ }^{5}$, Bloom ha revitalizado y sistematizado el estudio de las influencias. Pretendo aquí una aplicación detallada de lo que llama Bloom los revisionary ratios («relaciones de revisión»); tomo el caso de «Yo persigo una forma» para sugerir que el poema de Darío constituye una "deslectura» creativa del soneto de Mallarmé «Mes bouquins refermés sur le nom de Paphos». Así empiezo con lo que, a mi juicio, es la explicación más clara de la posición de Harold Bloom:

The first principle of the proper study of influence, as I conceive it, is that no strong poem has sources and no strong poem merely alludes to another poem. The meaning of a strong poem is another strong poem, a precursor's poem which is being misinterpreted, revised, corrected, evaded, twisted askew, made to suffer an inclination or bias which is the property of the later

3 Tzvetan Todorov, «Une complication du texte: les Illuminations», Les genres du discours, París, Seuil, 1978, p. 206.

4 Rubén Dario, Prosas profanas y otros poemas, edición de Ignacio M. Zuleta, Madrid, Clásicos Castalia, 1983, p. 40.

5 The Anxiety of Influence, New York and London, Oxford University Press, 1973 y A Map of Misreading, New York, Toronto and Melbourne, Oxford University Press, 1975. 
and not the earlier poet. Poetic influence, in this sense, is actually poetic misprision, a poet's taking or doing amiss of a parent-poem that keeps finding him, to use a Coleridgean turn-of-phrase. Yet even this misprision is only the first step that a new poet takes when he advances from the early phase where his precursor floods him, to a more Promethean phase where he quests for his own fire, which nevertheless must be stolen from his precursor. I count some half-dozen steps in the life-cycle of the strong poet, as he attempts to convert his inheritance into what will aid him without inhibiting him by the anxiety of a failure in priority, a failure to have begotten himself. These steps are revisionary ratios, and for the convenience of shorthand, I find myself giving them arbitrary names, which are proving useful to me, and perhaps can be of use to others. ${ }^{6}$

Las seis relaciones, ya clásicas para bien o para mal, de acuerdo con la opinión que uno tenga de Bloom, son: Clinamen, Tessera, Kenosis, Demonización, Askesis y Apófrades. Daré en su momento cada una de las definiciones de Bloom añadiendo sólo que las voy a aplicar en orden diferente, siguiendo el desarrollo del soneto de Darío. Así, he planteado mi consideración de las relaciones de Bloom según ocurren en una lectura lineal de «Yo persigo una forma», hecha en yuxtaposición con «Mes bouquins refermés sur le nom de Paphos».

Mes bouquins refermés sur le nom de Paphos, il m'amuse d'élire avec le seul génie une ruine, par mille écumes bénie sous l'hyacinthe, au loin, de ses jours triomphaux.

Coure le froid avec ses silences de faux, je n'y hululerai pas de vide nénie si ce très blanc ébat au ras du sol dénie A tout site l'honneur du paysage faux.

Ma faim qui d'aucuns fruits ici ne se régale trouve en leur docte manque une saveur égale: qu'un éclate de chair humain et parfumant!

Le pied sur quelque guivre où notre amour tisonne, je pense plus longtemps, peut-être éperdument, de l'autre, au sein brûlé d'une antique amazone ${ }^{7}$.

Yo persigo una forma que no encuentra mi estilo, botón de pensamiento que busca ser la rosa; se anuncia con un beso que en mis labios se posa al abrazo imposible de la Venus de Milo.

6 Bloom, Figures of Capable Imagination, pp. 9-10. Ver aquí también la definición de «Clinamen», «Tessera», «Kenosis», «Daemonization», «Askesis»y «Apophrades».

7 Stéphane Mallarmé, Oeuvres complètes, París, Pléiade, 1945, p. 76. 
Adornan verdes palmas el blanco peristilo;

los astros me han predicho la visión de la Diosa;

y en mi alma reposa la luz como reposa

el ave de la luna sobre un lago tranquilo.

$Y$ no hallo sino la palabra que huye,

la iniciación melódica que de la flauta fluye

y la barca del sueño que en el espacio boga;

y bajo la ventana de mi Bella Durmiente,

el sollozo continuo del chorro de la fuente

y el cuello del gran cisne blanco que me interroga ${ }^{8}$.

Clinamen, which is poetic misprision proper; I take the word from Lucretius, where it means a swerve of the atoms so as to make change possible in the universe. The later poet swerves away from the precursor, by so reading the parent-poem as to execute a clinamen in relation to it. This appears as the corrective movement of his own poem, which implies that the precursor poem went accurately up to a certain point, but then should have swerved, precisely in the direction that the new poem moves.

Los dos sonetos, siendo los últimos poemas de sus respectivos volúmenes, constituyen un momento de «bouquins refermés». El poema de Mallarmé hace eco del cierre de su propia colección en el brusco cierre de su primer verso. El apelativo bajo (algo despectivo) de «bouquins», además, sólo subraya la falta relativa de importancia de Poésies, ese libro que podía leerse junto al fuego, en el contexto del proyecto-sueño de Mallarme de El Libro («Le Livre, instrument spirituel») ${ }^{9}$.

La insatisfacción de Mallarmé para con la literatura tradicional —los meros «bouquins» reminiscentes del Verlaine de «Et tout le reste est littérature ${ }^{10}$ - le permite no obstante visualizar lo que dicha literatura podría alcanzar: «Je crois que la littérature [...] nous fournira un Théâtre, dont les représentations seront le vrai culte moderne; un Livre explication de l'homme suffisante à nos plus beaux rêves»" ${ }^{11}$. Es mi intención demostrar que la brecha que existe, para Mallarmé, entre su propio estilo, en Poésies, y la forma de su sueño-proyecto constituye el clinamen de «Yo persigo una forma». Por lo tanto, es necesario considerar

8 Prosas profanas y otros poemas, ed. Zuleta, p. 177.

9 Ver Quant au livre, Pléiade, pp. 369-387.

10 Paul Verlaine, "Art poétique», Oeuvres complètes, París, Pléiade, 1968, p. 326.

11 Plëiade, pp. 875-6. 
primero cómo persigue el propio Mallarmé una forma adecuada de la expresión poética.

La dificultad de cualquier lectura o relectura de la obra de Mallarmé debe subrayarse de antemano porque toda propuesta respecto de las misprisions de Darío tiene que ser situada en el contexto de las famosas faltas provocadas de continuidad en pensamiento y expresión que caracterizan la obra de Mallarmé. Entre los muchos críticos que han tratado este tema, es tal vez Malcolm Bowie el que mejor resume el problema en su libro Mallarmé and the Art of Being Difficult ${ }^{12}$ :

The double effort required to allow Mallarmés gaps the full disjunctive and destructive power, yet at the same time remain attentive to the multitude of invisible currents which pass back and forth between the separated segments, will strike many readers as inexcusably arduous and unrewarding [...] such moments are of the essence in Mallarmé [...] the type of modern artist [...] intent on breaking up ready-made Gestalten and smooth surface textures in order to compel his audience to look elsewhere for artistic coherence, to venture beneath the surface into the difficult, undifferentiated world of unconscious process, to interrupt the easy flow of horizontal perception with strenuous excursions into multi-level, all at once verticality ${ }^{13}$.

Si la fórmula más precisa de la estética del Simbolismo es la de Mallarmé, «peindre non la chose mais l'effet qu'elle produit» ${ }^{14}$, entonces el cierre de la literatura «sur le nom de Paphos», al menos aquí, ofrece un ejemplo eficaz de la forma sugerida por tal estética: «Nommer un objet, c'est supprimer les trois quarts de la jouissance du poème, qui est faite de deviner peu à peu: le suggérer, voila le rêve. C'est le parfait usage de ce mystère qui constitue le symbole» ${ }^{15}$. Es decir, que el hecho de terminar la lectura en el mismo nombre de Paphos desvía la atención de cualquier hecho histórico, de toda asociación mítica. Lejos de meditar o reformular las ideas evocadas por la mención del lugar sagrado de Afrodita en Chipre, fundado por las Amazonas, el soneto lucha contra aquel «flujo fácil de percepción horizontal», como sugiere Bo-

12 Malcolm Bowie, Mallarmé and the Art of Being Difficult, Cambridge, Cambridge University Press, 1978.

13 Bowie p. 8 y p. 16. Bowie se refiere aquí al estudio de Anton Ehrenzweig, The Hidden Order of Art, London, Weidenfeld \& Nicholson, 1967.

14 «Lettre à Henri Cazalis» de octubre-noviembre 1894, Correspondance, Vol. 1, 1862-71, edición del Henri Mondor y Jean-Pierre Richard, París, Gallimard, 1959, p. 137.

15 Pléiade p. 869. 
wie, intentando en sus cuartetos precisamente esas «excursiones vigorosas» de una proposición imaginativa creada sólo para ser negada, de una visión de la gloria del pasado evocada sólo para ser condicionalmente descubierta como un engaño por la corriente de aire frío que obstaculiza una lectura hogareña. Mientras proceden los cuartetos, de manera disyuntiva, por intermedio de negaciones hasta le faux /lo falso -y por lo tanto subrayando incluso la imposibilidad de un acceso a un «paysage» históricamente irrecuperable - los tercetos construyen precisamente esa «verticalidad múltiple y simultánea» de Mallarmé, saboreando una estética de la ausencia convocada, de un modo que no es más explícito en ningún otro poema:

Je dis: une fleur! et, hors de l'oubli où ma voix relègue aucun contour, en tant que quelque chose d'autre que les calices sus, musicalment se lève, idée même et suave, l'absente de tous bouquets ${ }^{16}$.

Así que el clinamen ocurre en «Yo persigo una forma» contra una estética de la prioridad de la palabra sobre la idea, de la ausencia convocada sobre la presencia física o hasta metafísica.

El soneto de Darío sigue el modelo del de Mallarmé, al cerrar un «bouquin» (Prosas profanas) con una nota de insuficiencia. Lo hace sin embargo con el deseo correctivo de modificar la estética simbolista o desviarse de ella. Mientras Mallarmé juega con la tradicional distinción contenido/forma, por la cual la forma ha abolido el contenido antes de ser abolida por la abstracción «ausencia», Darío añade a la existente dicotomía un tercer elemento, a saber, el «estilo». Mientras el poema de Mallarmé tiene como objetivo la destrucción irónica de la noción de que la forma poética puede expresar un contenido como presencia, el soneto de Darío, en su primer verso, re-establece la forma como objetivo de aquella búsqueda constantemente negada, frustrada sólo por lo inadecuado de un estilo personal. La ausencia, por consiguiente, no constituye una condición necesaria, ni mucho menos el objetivo mismo, porque el soneto de Darío implica ya, desde su primer verso, la persecución de una trascendencia que, cada vez más en su poesía posterior, asumirá la forma de un logocentrismo desesperado, por no decir una total teleología ética. En esta fase de su producción literaria, sin embargo, «Yo persigo una forma» se desvía de la noción mallarmeana de que «ce n'est pas avec des ideés qu'on fait des sonnets [...] mais avec

16 Pléiade p. 857. 
des mots» ${ }^{17}$, cuando re-abre su propio libro con un pensamiento: que de una posible fusión de estilo y forma, puede tal vez florecer un ideal artístico hasta entonces inalcanzado. Al respecto, claro, es Darío heredero de aquellas generaciones anteriores de románticos y parnasianos y, aun más quizá, del ideal wagneriano del Gesamtkunstwerk, como demostrarán ciertos elementos posteriores del soneto ${ }^{18}$. En resumen, y en términos del análisis bloomiano, Mallarmé ha absorbido tal herencia adecuadamente hasta cierto punto, pero luego debería haberse desviado precisamente en el sentido del nuevo poema. Parecería, a primera vista, que Darío es incapaz de aceptar ni la trayectoria ni las implicaciones no-teológicas de la estética mallarmeana, percibiendo ya quizá, a tan corto plazo, lo que propone Malcolm Bowie tres cuartos de siglo después:

Mallarmé occupies a special place in the modern tradition. Among French poets of the nineteenth century he was the most adventurously and the most trenchantly agnostic: his powers of doubt played not only upon the timehonoured theologies and theodicies of Europe, but upon those new, secular cults of beauty and «the Spirit» of which he is popularly thought to be an uncritical exponent ${ }^{19}$.

Para un autor que dentro de muy pocos años escribiría del Arte «Ego sum lux et veritas et vita», la intuición de Mallarmé podía difícilmente ofrecer algún consuelo.

Tessera, which is completion and antithesis; I take the word not from mosaic-making, where it is still used, but from the ancient Mystery-cults, where it meant a token of recognition, the fragment, say, of a small pot which with the other fragments would reconstitute the vessel. The later poet antithetically «completes» the precursor, by so reading the parent-poem as to retain its terms but to mean them in an opposite sense, as though the precursor had failed to go far enough.

Los términos retenidos en el poema de Darío pero que significan en sentido opuesto son términos de ausencia: «el abrazo imposible».

17 Henri Mondor, Vie de Mallarmé, París, Gallimard, 1941, p. 684. Ver también «L'oeuvre pure implique la disparition élocutoire du poète, que cède l'initiative aux mots», Pléiade, p. 36.

18 Ver también: «Ya nos conduzca sobre esas grandes alas arcangélicas hacia el universo wagneriano; a su concepción ideal de la Representación; a la futura realización del Libro cósmico de arte», Rubén Darío, Obras completas, Tomo IV, Cuentas y novelas, Madrid, Afrodisio Aguado, 1955, p. 920.

19 Bowie, p. 4. 
El sentido, sin embargo, es el de incumplimiento, de remordimiento, a diferencia de la no-presencia convocada, o más bien la no-presencia deseada de «sein brûlé». Es decir, el clinamen de Darío consiste en su incapacidad o poca voluntad de aceptar le docte, núcleo de cerebralidad mallarmeana que iguala («égale») la ausencia y la presencia. La aspiración de Darío reúne lo estético con lo sensual en su primer cuarteto (aunque, como se puede ver con relación a apófrades, retiene, inseparablemente, un elemento de lo cerebral en el doble sentido de pensamiento). De manera crucial, su soneto está construido no sobre un «término ausente» ${ }^{20}$, como en el caso del «rêve» de Mallarmé, sino sobre «objetivos correlativos» de su propio «rêve», tan concretos, por ejemplo, como «la barca del sueño». Mallarmé se evade de «l'ici» en su trayecto hacia «manque»; Darío nunca abandona ni «l'ici», ni «l'ici-bas». Un arquetipo romántico de la evasión marina (lo real) se mezcla siempre con una evasión mental simbolista (lo irreal).

En este caso, tessera significa no tanto que «el precursor no haya avanzado lo suficiente», sino que el soneto de Mallarmé avanzó demasiado e inaceptablemente lejos, elevando lo erudito y lo cerebral, quizá incluso lo espiritual, sobre y más allá de la igualmente importante hambre («faim») de Darío por los frutos de la sensualidad. La hiperestesia, lejos de ser alcanzable a través de una lectura doméstica y hogareña de butaca - de modo divertido («il m'amuse») y a voluntad («élire»)— se persigue, en el caso de Darío, por medio de una iconografía totalmente distinta, la de la sinestesia - «rosa», «beso», «labios», «se anuncia» y «abrazo»-, así sea inalcanzablemente.

Daemonization, or a movement toward a personalized Counter-Sublime, in reaction to the precursor's Sublime; I take the term from general NeoPlatonic usage, where an intermediary being, neither Divine nor human, enters into the adept to aid him. The later poet opens himself to what he believes to be a power in the parent-poem that does not belong to the parent proper, but to a range of being just beyond that precursor. He does this, in his poem, by so stationing its relation to the parent-poem as to generalize away the uniqueness of the earlier work.

La noción de «terme absent» tiene cierta relación, sobre todo en el contexto del juego présence/absence, con Derrida. En términos técnicos, sin embargo, «le rêve» (como «le terme absent») se refiere directamente a la teoría de «repressed matrix» de Michael Riffaterre, Semiotics of Poetry, London, Methuen, 1978, p. 19. Ver también mi «Undoing the Romantic Discourse: A Case-Study in Post-Structuralist Analysis. Vallejo's Trilce I», Romance Studies, University of Wales, No.5, 1984-5, pp. 91-111. 
Lo anti-sublime personalizado de Darío proviene de un movimiento hacia la visión de una forma específica de la deidad: «Los astros me han predicho la visión de la Diosa».

Como reacción a lo sublime del precursor ( «Je pense plus longtemps, peut-être éperdument, / à l'autre, au sein brûlé d'une antique amazone), Darío se abre al poder del (de lo) otro no limitado a un ensueño personal del seno ausente sino a una gama del ser más allá de aquella visión. Su anti-sublimidad es a la vez más cósmica («los astros») y más tradicional («la Diosa»), aunque queda un fuerte sentido de lo «personalizado» en el privilegio concedido a un poeta/vidente elegido por el destino («me han predicho»).

Darío generaliza lo que tiene de único la obra anterior en tanto que la fusión de lo religioso, lo personal y lo fatalista parece llevar su soneto más allá de la mera «Beauté» del Simbolismo, reiniciando la búsqueda de lo Ideal rechazado por Mallarmé en una primera etapa de su obra $^{21}$. Pero, ies éste el caso? Reservaré mi respuesta a esta pregunta hasta mi consideración de kenosis. Quiero antes mostrar cómo la demonización funciona en «Yo persigo una forma».

El poder derivado de la evocación inicial mallarmeana de «Une ruine, par mille écumes bénie» invade también la construcción equivalente: «Adornan verdes palmas el blanco peristilo». Pero el soneto de Darío va más allá. El verso construye no únicamente un santuario impenetrable para la Diosa anhelada por Darío. Porque el follaje frondoso que a la vez adorna y entenebrece un peristilo arquitectónico (las columnas que circundan un templo) está constituido también, en verso, por el adorno esencialmente modernista del peri(frástico e)stilo de $\mathrm{Ru}$ bén Darío. «Peristilo», pues, evoca no sólo un contenido (externo) sino también una forma (interna), es decir, la de la estética modernista tan frecuentemente asociada con el Simbolismo pero que se puede comparar, con más exactitud (por concentrarse en el congelamiento de contenido y forma en «le bloc immobile de l'Art») con el Parnasse de Gautier. Tal como en el caso de «pensamiento», «peristilo» funciona paranomásticamente - como un juego de palabras-y lo hace (con)fundiendo dos presencias equivalentes. El concepto que resulta opera a un nivel más comparable con el conceptismo renacentista que con la técnica ma-

21 «Après avoir trouvé le Néant, j'ai trouvé le Beau», Correspondance, p. 220. 
llarmeana de yuxtaponer una presencia imaginada a una convocada ausencia.

Por penetrar así en un peristilo personal, el poema de Darío parece alcanzar, al terminar el segundo cuarteto, precisamente la «forma perseguida», «la gama del ser que se encuentra apenas más allá del precursor»:

Y en mi alma reposa la luz, como reposa el ave de la luna sobre un lago tranquilo.

Tal momento de suspensión, de reposo, de levitación cuasi-mística ante la potencial consumación y resolución del sexteto inminente, no se produce en la tradicional posesión del alma del místico por la paloma del Espíritu Santo, el Paráclito, sino en su equivalente quintaesencialmente modernista a saber, el Cisne, aunque indirectamente evocado, encarnación Ideal de lo espiritual, del Arte. Queda aún por verse, no obstante, si los tercetos del soneto sostendrán y cumplirán las expectativas creadas en sus cuartetos, es decir, la posibilidad de unir «estilo» y «forma», de alcanzar el Ideal explícitamente rechazado por el cuartetoprecursor mallarmeano:

Je n'y hululerai pas de vide nénie

si ce très blanc ébat au ras du sol dénie

à tout site l'honneur du paysage faux.

El triunfo proviene aquí de una construcción de negatividades las partículas verbales negativas n'y/pas/ne/nie/dénie- que constituyen en sí mismas las columnas entenebrecedoras, el peristilo específicamente mallarmeano que circunda un vacío consagrado. A este respecto, diría yo que el poema de Mallarmé contiene su propia anti-sublimidad, su propia demonización, como reacción contra lo que el poema de Darío, hasta este punto, en una misprision clásica, interpreta como el templo a lo sublime del precursor: en realidad, una presencia desmoronada, «une ruine».

Kenosis, which is a breaking-device similar to the defense mechanisms our psyches employ against repetition -compulsions; kenosis, then, is a movement toward discontinuity with the precursor. I take the word from St Paul, where it means the humbling or emptying-out of Jesus by himself, when he accepts reduction from Divine to human status. The later poet, apparently emptying himself of his own afflatus, his imaginative godhood, seems to humble himself as though he ceased to be a poet, but this ebbing is so performed in relation to a precursor's poem-of-ebbing that the precursor is emptied out also, and so the later poem of deflation is not as absolute as it seems. 
Una re-iniciación de la búsqueda de lo Ideal, como posible alternativa a la ausencia docta («docte») que saborea el precursor, como he sugerido antes, se resuelve no solamente a la manera tradicional del soneto, en el sexteto, sino también en la relación de revisión bloomiana de kenosis. El «vaciarse de su propia inspiración», el humillarse de la aspiración de Darío asume la forma de «Y no hallo sino...», que se opone a «Yo persigo...». Lo que sí es alcanzable, de manera provocadoramente insuficiente, es «la palabra». Aquí huye la palabra mientras, en el poema de Mallarmé, huye la Idea: dejando que la Palabra —en este caso «Paphos»- siga haciendo eco bien después del cierre del libro, privada de todo contexto de presencia, abandonada al mero juego de resonancias, un cabrilleo («l'ébat») de garantizada ausencia.

Es cierto, sin embargo, que el poema posterior contiene «una técnica de ruptura semejante a los mecanismos de defensa que nuestras psiques emplean». Por una parte, existe una aspiración a un estado inefable similar al de la música habitualmente concedida a la Palabra por generaciones de posrománticos e, irónicamente, no menos por Mallarmé: «Je sais, on veut à la Musique, limiter le mystère; quand l'écrit y prétend $\gg{ }^{22}$.

La «iniciación melódica» sirve aquí como una consolación anticipatoria a una instancia más del fracaso, la última incapacidad de unir «estilo» y «forma» al final de Prosas profanas. El poema de Darío se aferra al imperativo de Verlaine - «de la musique avant toute chose»del mismo modo que su estética va a desarrollarse más de acuerdo con la Sagesse de este último que con el método no tan obviamente ético (aunque infinitamente más revolucionario) de Un coup de dés...

Por otra parte, este poema posterior de deflación no es tan absoluto como parece, ni podría serlo. Porque el mecanismo de defensa mostrado anteriormente en el contexto estético opera también el nivel ético. "Y no hallo sino [...] la barca del sueño que en el espacio boga», constituye apenas una media kenosis, y no consigue abandonar el eco de suspensión e iluminación potencial del segundo cuarteto analizado con relación a la demonización. Nostálgica, sin embargo, por aquel estado perdido de tranquilidad receptiva, aquella disponibilidad por una trascendencia cuasi-mística, la visión onírica evoca solamente un corre-

22 Pléiade, p. 385. 
lativo de evasión que se resuelve en lugar común -a saber, la evasión marina y no la mental - mencionado ya cuando consideré tessera, e identificado como indeleblemente romántico.

En resumen, entonces, aunque kenosis funciona aquí como «un movimiento hacia una discontinuidad con el precursor», cabe preguntarse si el «humillarse» o «vaciarse» que ocurre en el primer terceto del soneto de Darío se ejerce en relación con «Mes bouquins refermés» de tal manera que apenas indica la poca voluntad, posiblemente la incapacidad de Darío de aceptar que Mallarmé rehuse llorar, con un lamento vacío («nénie»), la pérdida de una trascendencia escapista por medio del Arte visto como un vehículo hacia lo Ideal.

Askesis, or a movement of self -purgation which intends the attainment of a state of solitude; I take the term, general as it is, particularly from the practice of pre-Socratic shamans like Empedocles. The later poet does not, as in kenosis, undergo a revisionary movement of emptying, but of curtailing; he yields up part of his own imaginative endowment, so as to separate himself from others, including the precursor, and he does this in his poem by so stationing it in regard to the parent poem as to make that poem undergo an askesis also; the precursor's endowment is also truncated.

El último terceto del soneto de Darío incorpora «un movimiento de auto-purgación» y el logro de «un estado de soledad». Al desplazar el «sollozo» de la persona a la naturaleza, el poema explota una falacia patética asociada arquetípicamente al Romanticismo, pero lo hace, en este caso, en un contexto específicamente modernista. Lo doméstico de la meditación hogareña de Mallarmé encuentra un paralelo en la muy diferente contemplación, en el jardín, de un potencial príncipe salvador de la Bella Durmiente. Este estado de soledad, por lo tanto, «cede una parte del legado imaginativo» de Darío tanto porque evoca una tradición de cuento de hadas como porque difunde la emoción personal en una naturaleza complaciente y dócil.

La figura principal de esta difusión es una de las más celebradas imágenes de Darío: «el cuello del gran cisne blanco que me interroga». Este verso encarna la duda implícita de una ética romántica en una iconografía de la fijeza Parnasiana. Casi análoga al Modernismo, la imagen del cisne en una interrogación helada, diría yo, difiere del modelo de Simbolismo mallarmeano precisamente porque re-formula su clásico decir: «peindre non la chose mais l'effet qu'elle produit». En una palabra, la poesía de Darío (su «estilo») está condenada a «peindre la chose et l'effet qu'elle produit», dando lugar a una insatisfacción per- 
petua en su persecución de «una forma»: lo inefable.

La cuestión de si se cumple la segunda condición de askesis es problemática. Podría argüirse que el legado de Mallarmé, la herencia del Simbolismo tal como es entendida inadecuadamente por muchos críticos, y tal vez por el mismo Darío, se ve limitada. Es decir, que una serie de imágenes - «croisée», «miroir», «vol», «cygne»y «azur»- ha constituido la fuerte pero malversada influencia de Mallarmé sobre sus sucesores. En una palabra, una iconografía de lo inalcanzable ha ocultado y suplantado la metodología para convocar la ausencia.

De modo inevitable, la explicación es tan teológica como sugiere la palabra iconografía. Porque askesis, se debe tener presente, proviene de la práctica de los shamanes presocráticos. He sugerido antes, en mi consideración de clinamen, que Darío parecía inicialmente incapaz de aceptar la trayectoria no-teológica de Mallarmé. El último terceto de «Yo persigo una forma» confirma este hecho. Pero este poema no es el único ejemplo de una lectura revisionista de Mallarmé por parte de Darío; y los ecos son elocuentes:

Ausencia de una religión; presencia virtual de todas, en su relación con el misterio, y las pompas litúrgicas, virtud de los signos, secreta fuerza de las palabras; el ensalmo musical; lo hierático en movimiento... he aquí que traza un signo nuevo, sobre el lago en silencio, el Cisne que comprende ${ }^{23}$.

Esta valoración última de Mallarmé por parte de Darío, su necrología, me conduce a la sexta y última de las relaciones de Harold Bloom.

Apophrades, or the return of the dead; I take the word from the Athenian dismal or unlucky days upon which the dead returned to reinhabit the houses in which they had lived. The later poet, in his own final phase, already burdened by an imaginative solitude that is almost solipsism, holds his own poem so open again to the precursor's work that at first we might believe the wheel has come full circle, and that we are back in the later poet's flooded apprenticeship, before his strength began to assert itself in the revisionary ratios of clinamen and the others. But the poem is now held open to the precursor, where once it was open, and the uncanny effect is that the new poem's achievement makes it seem to us, not as though the precursor were writing it, but as though the later poet himself had written the precursor's characteristic work.

A estas alturas, es posiblemente claro que la tesis de este estudio acerca de la respuesta ( $¿ 0$ «responso»?) de Darío a Mallarmé es que el 
poeta posterior ya cargado lleva a cabo una deslectura retadora del precursor. Darío opta por leer a Mallarmé como le Cygne más que como le signe.

En «Yo persigo una forma», Darío re-abre su poema desde el primer cuarteto a la obra de Mallarmé. La insuficiencia de su propio estilo promueve la persecución de una influencia mayor del «aprendizaje inundado del poeta posterior, antes de que su fuerza empezara a afirmarse en las relaciones revisionarias de climamen y las demás». Así, inicialmente, el «botón de pensamiento que busca ser la rosa» de Darío estaba abierto, de manera humilde y relacionada con kenosis, a la fórmula de Mallarmé «Je dis: une fleur...». Visto de un modo retrospectivo, según la aplicación de las relaciones de Bloom, el poema de Darío se reabre al «efecto inaudito» de alcanzar «lo otro» momentáneamente, por su capacidad de evocar el poema de Mallarmé en una lectura revisionista o en una deslectura.

Dos transformaciones están en juego. Primero, la humilde flor (pensamiento) que aspira a la belleza consumada de la rosa; segundo, la igualmente humilde, embriónica idea (pensamiento) que se abre osadamente, pero en un último fracaso, al logro monumental mallarmeano de «l'absente de tous bouquets». El soneto de Darío empieza con la misma nota de «Je pense...» con la cual termina el de Mallarmé. El soneto no puede, sin embargo, arriesgar «éperdument»-distraidamente, es decir, tanto en la alegría como en la locura - las implicaciones de aquella estética de consumación no alcanzable aquí («ici»), ni en cualquier pasión física («où notre amour tisonne»). Aunque trasciende la domesticidad mallarmeana (¿el matrimonio?), el coqueteo de Darío con las míticas «Diosa» y «Bella Durmiente» subraya su apenas parcial reescritura de «la obra característica del precursor». Ésta apenas desplaza el objeto amado y en ello alude a lo románticamente inalcanzable; el soneto no es capaz de optar por el espacio vacío que resulta de la flameante no-consumación de «l'autre (amour)», el «sein brûlé» de un mito inexistente. Si el poema de Darío lleva más lejos su herencia mallarmeana, apenas re-inscribe una interrogación en «le vide papier que la blancheur défend». Pero esto no es una condena, porque Darío no es el único que siente malestar hacia la invitación mallarmeana al silencio.

No ha sido mi intención en este estudio generalizar ni extender las lecciones del análisis bloomiano al dominio de la historia literaria. Mi preocupación no es tanto si Darío es parnasiano o simbolista como mos- 
trar el modo en que la influencia poética funciona técnicamente. Por esta razón he querido concentrarme más en el juego intrincado de las relaciones de Harold Bloom que en la especulación psicoanalítica en la cual ocurren. No he perseguido a Darío como un caso de poeta viviendo ansiosamente a la sombra de Mallarmé, mucho menos como alguien encerrado en una rivalidad edípica con un precursor castrador. Simplemente he partido de otro de los prinicipios de Bloom: la noción de que los poemas existen primordialmente contra tres adversarios: 1) ellos mismos, 2) otros poemas, 3) el tiempo ${ }^{24}$. Yo propondría un paralelo a este trío en términos de metodología crítica, como sigue: 1) la crítica textual, 2) la intertextualidad, 3) el estudio de las influencias. Mi objetivo en esta yuxtaposición de "Yo persigo una forma» y «Mes bouquins refermés» ha sido poner en funcionamiento, inseparablemente, las tres.

24 Ver el estudio de Roger Poole sobre el contexto de la crítica psicoanalítica de Harold Bloom, «The Yale School as a Theological Enterprise», Structuralisms, Renaissance and Modern Studies, XXVII, 1983, p. 24. 